\section{ORIGINAL RESEARCH}

N. Lummel

C. Zeif

A. Kloetzer

J. Linn

H. Brückmann

H. Bitterling

\title{
Variability of Morphology and Signal Intensity of Alar Ligaments in Healthy Volunteers Using MR Imaging
}

\begin{abstract}
BACKGROUND AND PURPOSE: Evaluation of alar traumatic injuries by using MR imaging is frequently performed. This study investigates the variability of morphology and signal intensity of alar ligaments in healthy volunteers so that pathology can be more accurately defined.
\end{abstract}

\begin{abstract}
MATERIALS AND METHODS: Fifty healthy volunteers were examined on a 1.5T MR imaging scanner with 2-mm PD-weighted sequences in 3 planes. Delineation of the alar ligaments in 3 planes and signal-intensity characteristics on sagittal planes were analyzed by using a 4-point grading scale. Variability of courses and morphologic characteristics were described.
\end{abstract}

RESULTS: Delineation of alar ligaments was best viewed in the coronal plane, followed by the sagittal and axial planes. In the sagittal view, $6.5 \%$ of alar ligaments appeared homogeneously dark. Hyperintense signal intensity in up to one-third of the cross-sectional area was present in $33 \%$ of cases; in up to two-thirds of the cross-sectional area, in $45 \%$ of cases; and in more than two-thirds of the cross-sectional area, in $15 \%$ of cases. Of alar ligaments, $58.5 \%$ ascended laterally, $40.5 \%$ ran horizontally, and $1 \%$ descended laterally. The cross-sectional area was round in $41.5 \%$, oval in $51.5 \%$, and winglike in $6.5 \%$.

concLusions: On 1.5T MR imaging, the alar ligaments can be delineated best in the coronal and sagittal planes. Our data indicate a remarkable variability of morphology and course as well as signal intensity. This finding is contradictory to former publications assigning such alterations exclusively to patients with trauma.

ABBREVIATIONS: $\mathrm{AL}=$ alar ligaments; $\mathrm{PD}=$ proton-density; $\mathrm{SE}=$ standard error; $\mathrm{WAD}=$ whiplash-associated disorder

$\mathbf{T}$

he upper cervical ligaments are of crucial importance for movement and stability in the craniocervical spine. The alar ligaments connect the dens axis to the occipital condyles and have an important role in rotation as well as in anterior, posterior, and lateral flexion in the upper cervical spine. ${ }^{1-3}$ It is evident that lesions of the alar ligaments occur in the context of severe neck trauma. ${ }^{4-6}$ Nevertheless, the involvement of the alar ligaments in WAD has not been conclusively established in prior imaging and postmortem studies. ${ }^{7}$ Imaging studies showed that the upper cervical ligaments can be evaluated by using MR imaging, ${ }^{8-10}$ especially proton-weighted sequences. MR imaging studies analyzing alar ligaments have included not only noninjured individuals ${ }^{9-15}$ but also selected patients with WAD. ${ }^{8,11}$

"WAD" is defined as an injury caused by hyperextension and subsequent hyperflexion of the cervical spine associated with sudden acceleration and/or deceleration of the head, most often caused by a car crash. ${ }^{16}$ Whiplash injury of the cervical spine is a frequent issue requiring medical expertise. The consequential costs for automobile insurance companies amounted to approximately 500 million euro in Germany and 4.5 billion dollars in the United States in the late 1990s. ${ }^{17,18}$ Some authors claim that posttraumatic changes can be de-

Received May 2, 2010; accepted after revision June 9.

From the Department of Neuroradiology, University of Munich, Munich, Germany.

Please address correspondence to: Nina Lummel, MD, Department of Neuroradiology, University of Munich, Grosshadern, Marchioninistr 15, 81377 Munich, Germany; e-mail: nina.lummel@med.uni-muenchen.de

DOI 10.3174/ajnr.A2255 tected as high signal intensity of the alar ligaments on MR imaging and claim that these findings correlate with clinical symptoms. However, until now, results are inconsistent as to whether high signal intensity within the alar ligaments was more frequent in patients with WAD than in noninjured controls ( for $^{15}$; against ${ }^{9,11,13}$ ).

In this investigation, we systematically assessed the varying MR imaging appearances of the alar ligaments in a large sample size of healthy volunteers. The objective was to define pathologies of the alar ligaments more accurately to minimize misinterpretation of their structural and signalintensity variability as posttraumatic alterations in patients with WAD.

\section{Materials and Methods}

\section{Subjects}

The study was approved by the regional ethics committee. Fifty healthy volunteers ( 23 men, 27 women) who had provided their written informed consent were included. The average age of patients included in the study was 24.1 years (range, 20-31 years). A standard questionnaire was filled out by each volunteer before the examination. None of the volunteers had ever been involved in any car crash or sustained any head or neck trauma, as stated in their case histories. Volunteers with chronic neck pain with or without restricted mobility of the cervical spine were excluded. Furthermore, volunteers with malignant disease or disease with potential involvement of the cervical spine and craniocervical junction (eg, chronic polyarthritis, rheumatic arthritis, or juvenile arthritis) were excluded from the study. 


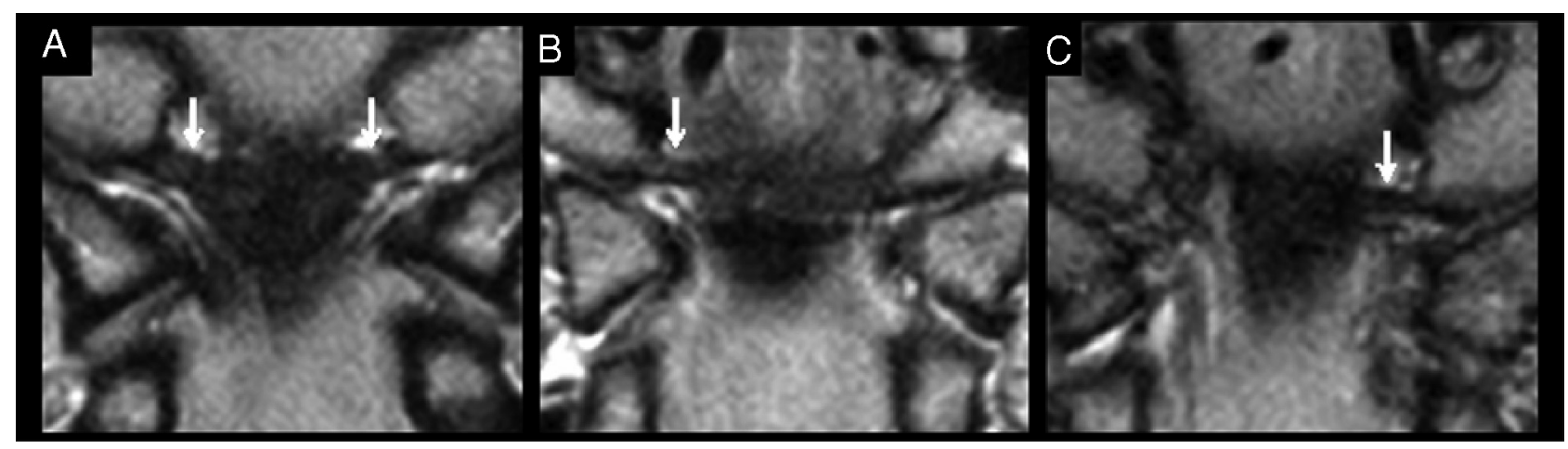

Fig 1. Proton attenuation-weighted MR images of alar ligaments (arrows) in the coronal plane demonstrate the variations in the course of the ligaments. Laterally ascending $(A)$, lateral $(B)$, and laterally descending $(C)$ orientations of the alar ligament are shown.

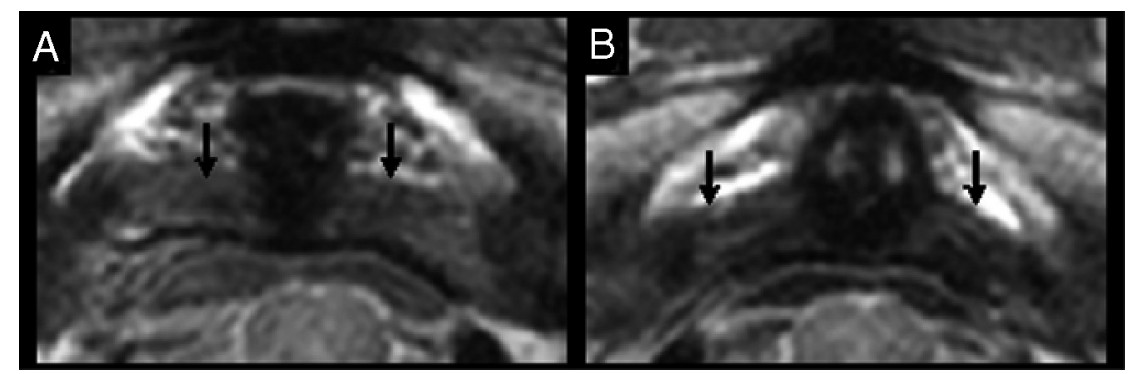

Fig 2. PD-weighted MR images of alar ligaments (black arrows) demonstrate their different orientations in the axial plane. Straight lateral $(A)$ and posterolateral $(B)$ orientations of the alar ligament are shown

\section{MR Imaging Protocol}

MR imaging was performed on a 1.5T scanner (Magnetom Vision or Symphony; Siemens, Erlangen, Germany). A standard head coil was used, and all images were obtained with the subject's head and neck in a neutral position. Proton-weighted and T2-weighted fast spin-echo images by using a double echo were obtained in 3 orthogonal planes. The imaging parameters were the following: TR, $3200 \mathrm{~ms}$; TE, $14 \mathrm{~ms}$ $(\mathrm{PD}) / 111 \mathrm{~ms}$ (T2); matrix, $192 \times 192 \mathrm{~mm}$; FOV, $120 \times 120 \mathrm{~mm}$; 2-mm section thickness; 19 sections.

\section{Image Evaluation}

Image interpretation was performed on a standard PACS workstation. All MR imaging examinations were independently evaluated by 2 neuroradiologists (with 9 and 3 years' experience with special training in head and neck imaging). One of these neuroradiologists reevaluated 20 randomly chosen cases (40 ligaments) in a second reading 4 weeks apart from the first one.

Only PD-weighted sequences were evaluated. Alar ligaments were characterized by the assessment of their delineation, orientation, shape, and signal intensity. Alar ligament delineation was evaluated in all 3 planes and classified into 4 groups: no (0), poor (1), moderate (2), and good (3) delineation. Orientation was specified in the coronal and axial planes. In the coronal view, ligaments were grouped into 3 categories: straight horizontal, laterally ascending, or descending orientation (Fig 1). Likewise, in the axial view, ligaments were divided into 3 groups: straight lateral, anterolateral, or posterolateral orientation (Fig 2). The shape was evaluated on sagittal images in the midportion of the ligament. According to prior studies, ligaments were grouped into 3 main variants: round, ovoid, and winglike (Fig 3). ${ }^{14}$ Furthermore, the signal intensity of alar ligaments was evaluated in the sagittal plane according to a 4-point grading scale proposed by Krakenes et al. ${ }^{15}$ Using this grading system on $\mathrm{PD}$ - weighted images, we graded low signal intensity throughout the entire cross-sectional area of the alar ligament as 0 , high signal intensity in less than onethird of the cross-section as 1 , high signal intensity in one-third to

\begin{tabular}{|c|c|c|}
\hline & Readers $1+2$ & $\kappa$ Value (SE) \\
\hline \multicolumn{3}{|l|}{ Delineation of $\mathrm{AL}$ in coronal plane } \\
\hline Good (grade 3) & $17 \%$ & \\
\hline Moderate (grade 2) & $50.5 \%$ & \\
\hline Poor (grade 1) & $32 \%$ & \\
\hline Not definable (grade 0) & $0.5 \%$ & \\
\hline \multicolumn{3}{|l|}{ Delineation of $\mathrm{AL}$ in sagittal plane } \\
\hline Good (grade 3) & $12 \%$ & \\
\hline Moderate (grade 2) & $40 \%$ & \\
\hline Poor (grade 1) & $47.5 \%$ & \\
\hline Not definable (grade 0) & $0.5 \%$ & \\
\hline \multicolumn{3}{|l|}{ Delineation of $\mathrm{AL}$ in axial plane } \\
\hline Good (grade 3) & $9.5 \%$ & \\
\hline Moderate (grade 2) & $41 \%$ & \\
\hline Poor (grade 1) & $46 \%$ & \\
\hline Not definable (grade 0) & $3.5 \%$ & \\
\hline Orientation of $\mathrm{AL}$ in coronal plane & & 0.85 \\
\hline Laterally ascending & $58.5 \%$ & $( \pm 0.051)$ \\
\hline Horizontally oriented & $40.5 \%$ & \\
\hline Laterally descending & $1 \%$ & \\
\hline Orientation of $\mathrm{AL}$ in axial plane & & 0.90 \\
\hline Posterolateral & $32.5 \%$ & $( \pm 0.047)$ \\
\hline Straight lateral & $67.5 \%$ & \\
\hline Anterolateral & $0 \%$ & \\
\hline \multicolumn{3}{|l|}{ Shape of AL in sagittal plane } \\
\hline Round & $41.5 \%$ & \\
\hline Ovoid & $51.5 \%$ & \\
\hline Winglike & $6.5 \%$ & \\
\hline Not definable & $0.5 \%$ & \\
\hline
\end{tabular}




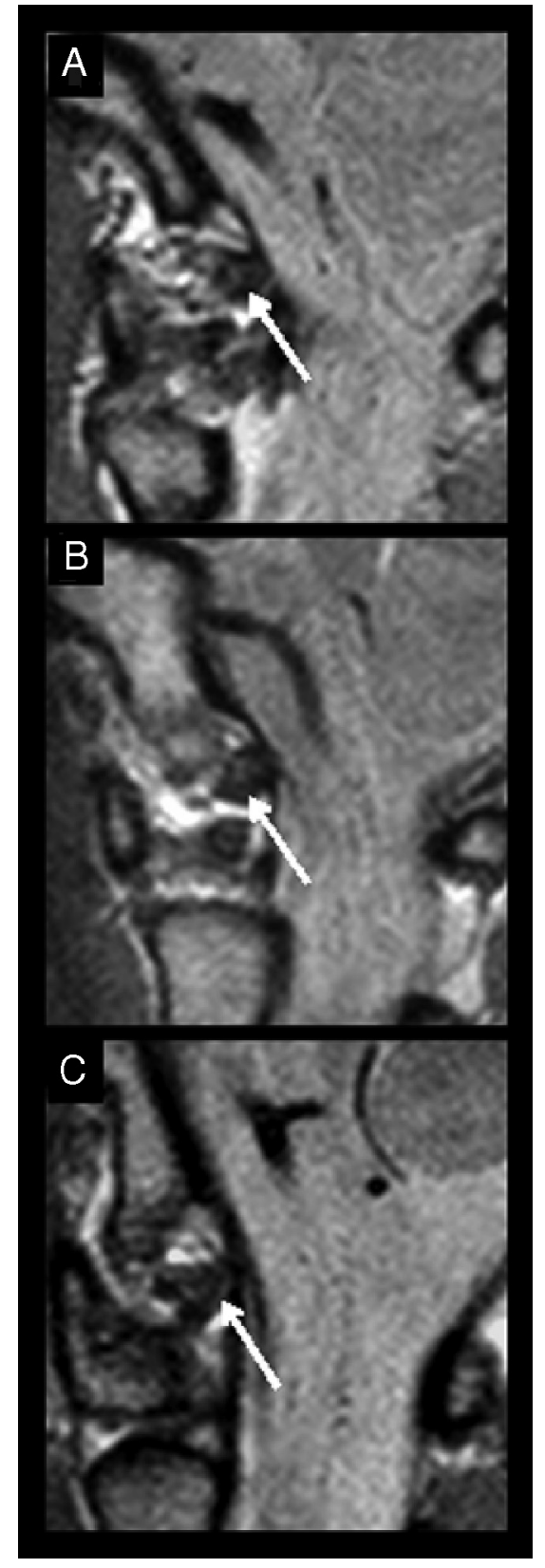

Fig 3. $P D-$ weighted $M R$ images of 3 alar ligaments (arrows) in the sagittal plane demonstrate the 3 different shapes in cross-section. $A$, Round ligament. $B$, Ovoid ligament. C, Winglike ligament.

two-thirds of the cross-section as 2, and high signal intensity in more than two-thirds of the cross-section as 3 (Fig 4).

\section{Statistics}

Cohen $\kappa$ coefficients $^{19}$ were used to evaluate interobserver agreements concerning orientation of alar ligaments in the coronal and axial views. Furthermore ordinary $\kappa$ coefficients were used to evaluate pair-wise interobserver agreement and intraobserver agreement concerning signal intensity. To replicate the analysis performed in prior studies, we dichotomized the 4-point scale for signal intensity into low signal intensity (grades 0 and 1 ) and high signal intensity (grades 2 and 3$){ }^{11,15,20}$

Statistical analyses were performed by using the Statistical Package for the Social Sciences, Version 16.0 (SPSS, Chicago, Illinois).

\section{Results}

Delineation of alar ligaments was best viewed in the coronal plane, with a moderate or good delineation in $67.5 \%$ of all images from the 50 volunteers (ie, 100 ligaments evaluated by 2 readers independently equaled 200 alar ligaments). Delineation was second-best in the sagittal plane with good-to-moderate delineation in 52\%. In the axial plane, the ligaments were of good or moderate delineation in $50.5 \%$. Details are given in Table 1.

On coronal images, ligaments were horizontally oriented in $40.5 \%$ and laterally ascending from the dens up to the condyles in $58.5 \%$ (Fig 1). Both readers found a laterally descending orientation in $1 \%$. In the axial view, a posterolateral orientation was found in $32.5 \%$, a straight lateral orientation was found in $67.5 \%$, and an anterolateral orientation was not found (Fig 2). Interobserver agreement for the orientation of alar ligaments in both coronal and axial views was very good $(\kappa=0.85 \pm 0.051 \mathrm{SE}$ for the coronal view; $\kappa=0.90 \pm 0.047 \mathrm{SE}$ for the axial view).

In cross-section, alar ligaments were grouped as round in $41.5 \%$, as ovoid in $51.5 \%$, and as winglike in $6.5 \%$ (Fig 3). One ligament was not definable in the sagittal view by 1 reader.

In the sagittal view, $6.5 \%$ of alar ligaments appeared homogeneously dark. A hyperintense signal intensity in up to onethird of the cross-sectional area was present in 33\% of cases; up to two-thirds of the cross-sectional area was hyperintense in $45 \%$ of the ligaments. Fifteen percent of the ligaments showed a hyperintense signal intensity in more than twothirds of the cross-sectional area (Table 2 and Fig 4).

As described in the "Materials and Methods" section, the groups were dichotomized into 2 categories to calculate interand intraobserver agreement concerning signal intensity: $39.5 \%$ as grade $0-1$, which belonged to group 1 ; and $60 \%$ as grade $2-3$, which constituted group 2. Subsequently, the $\kappa$ value for the interobserver agreement was 0.33 (SE of $\kappa \pm$ 0.090), indicating fair agreement.

The intraobserver agreement concerning signal intensity with respect to dichotomized groups was $\kappa=0.37$ (SE of $\kappa \pm$ 0.136), likewise indicating fair agreement.

\section{Discussion}

This study assessed the morphologic and signal-intensity variability of alar ligaments in an asymptomatic population by using MR imaging. Alar ligaments connect the lateral aspect of the dens and the medial inferior aspect of the occipital condyles. ${ }^{21}$ Because $\mathrm{PD}$-weighted images with $2-\mathrm{mm}$ section thickness without a gap emerged as the best protocol to image alar ligaments reliably, ${ }^{14}$ we applied this protocol in our study. Using PD - weighted sequences, Krakenes et al $^{14}$ characterized morphologic aspects of alar ligaments in vivo.

In terms of orientation of the ligaments, our results agree with prior studies in vitro ${ }^{2,22}$ and in vivo. ${ }^{9,14}$ Almost $60 \%$ of alar ligaments in our population showed a laterally ascending orientation, and approximately $40 \%$ were orientated horizontally. Descending orientation was rare. Likewise, in the axial plane, we found a lateral orientation in approximately onethird of cases and a posterolateral orientation in another third of cases. Anterolateral orientation was very rare. Furthermore, our results showed a high variability concerning the shape of the alar ligaments, also in accordance with the investigations 

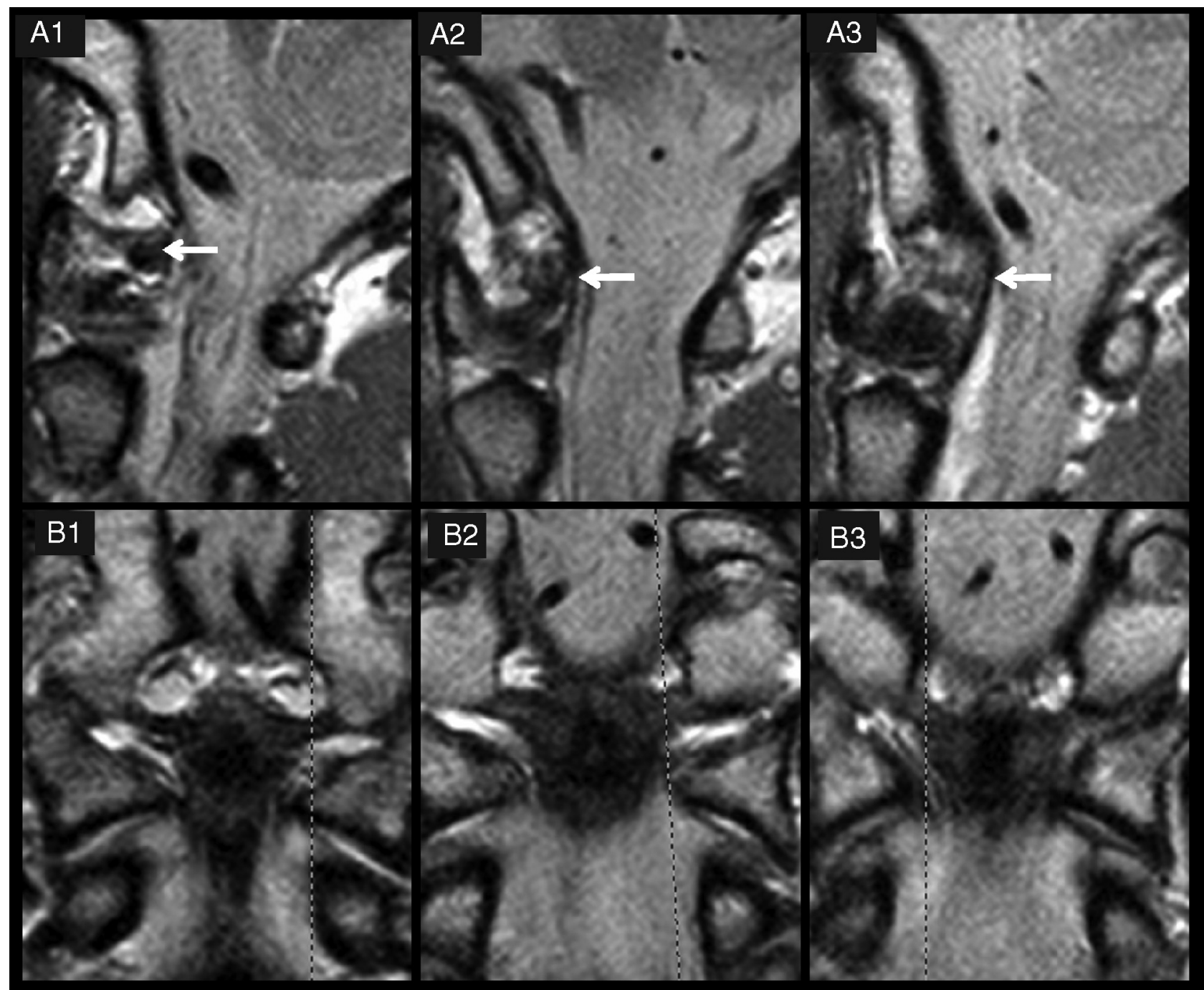

Fig 4. Three examples of grades of delineation and signal intensity of alar ligaments. $A$ and $B$, PD-weighted MR images of 3 alar ligaments in the sagittal $(A)$ and coronal $(B)$ planes The dashed lines in B1-3 indicate the orientation of the respective sagittal sections in $A 1-3$. In $A 1$, the alar ligament shows a good delineation (grade 3 ) and low signal intensity (grade 0 ). $A 2$ is classified as grade 2 for signal intensity (high signal intensity in one-third to two-thirds of the cross-section) and delineation (moderate). In $A 3$, the alar ligament is hardly definable (grade 1), with high signal intensity throughout the cross-section (grade 3).

\begin{tabular}{|c|c|c|}
\hline & $\begin{array}{l}\text { Reader } \\
1+2\end{array}$ & $\begin{array}{l}\kappa \text { Value } \\
\text { (SE) }\end{array}$ \\
\hline $\begin{array}{l}\text { Low signal throughout entire cross-section, } \\
\text { grade } 0\end{array}$ & $6.5 \%$ & $0.331 \pm 0.090$ \\
\hline $\begin{array}{l}\text { High signal in } \leq 1 / 3 \text { of cross-section, } \\
\text { grade } 1\end{array}$ & $33 \%$ & \\
\hline $\begin{array}{l}\text { High signal in } 1 / 3 \text { to } 2 / 3 \text { of cross-section, } \\
\text { grade } 2\end{array}$ & $45 \%$ & \\
\hline $\begin{array}{l}\text { High signal in } \geq 2 / 3 \text { of cross-section, } \\
\text { grade } 3\end{array}$ & $15 \%$ & \\
\hline Not definable & $0.5 \%$ & \\
\hline
\end{tabular}

mentioned before. In the cross-section, approximately onehalf appeared ovoid, slightly less than one-half appeared round, and the few remaining ligaments showed a winglike configuration.

In summary, considerable intra- and interindividual variations in orientation and shape of alar ligaments are a consistent finding. ${ }^{2,22}$ Hence, in the evaluation of alar ligaments (eg, in patients with WAD), an asymmetric or atypical appearance should not be misinterpreted as a pathologic finding.

In the literature, the significance of signal-intensity hyperintensities of alar ligaments as a sequela of WAD is inconsistent. Krakenes et al ${ }^{15}$ introduced a 4-point grading scale based on the signal intensity of alar ligaments in the sagittal plane. Low-signal intensity was defined as normal (grade 0 ). The authors reported high-signal-intensity changes (grade 2 or 3 ) only among patients with whiplash. Increased signal intensity (grades 1-3) was regarded as a sign of injury. On the other hand, Myran et $\mathrm{al}^{11}$ used the same MR imaging protocol, MR imaging equipment, and alar ligament grading scale as Krakenes et $\mathrm{al}^{14}$ but did not support the conclusion of Krakenes et al. Myran et al examined 3 different groups (1 group with persistent WAD grades I-II after a car crash, 1 group with chronic nontraumatic neck pain, and 1 group without neck pain or previous neck trauma) and found areas of high signal intensity (grades 2 and 3 ) in all 3 groups without significant differences between the groups. In our study, we also used a comparable MR imaging protocol and found sig- 
nal-intensity alterations (grades 2 and 3 ) in 60\% of alar ligaments in a group of healthy young volunteers without any history of WAD.

There are also previous reports of high-signal-intensity changes among asymptomatic individuals, ${ }^{9,13}$ but in these studies, different MR imaging protocols and different grading scales were used. Possible anatomic variances in the connective fiber attenuation may influence the imaging appearance of alar ligaments. Age-dependent degeneration and fatty tissue, interspersed among fibers, are also known to cause highsignal-intensity MR imaging changes in tendons and ligaments of other localizations. ${ }^{23-26}$ A similar effect could be conceivable for alar ligaments, even though a recent study could not demonstrate an association between the frequency of alar ligament hyperintensities and patients' ages. ${ }^{27}$ In 2008 , $\mathrm{Koch}^{28}$ examined specimens of the upper cervical spine after fatal head trauma pathoanatomically and histologically and by using MR imaging. Koch found significant inter- and intraindividual differences of interspersed fatty tissue in the alar ligaments and a respective correlation to the MR signal intensity of the ligaments.

Although taking into account the anatomic conditions of the structures that we analyzed (10- to $13-\mathrm{mm}$ length, $3 \times 6$ $\mathrm{mm}$ diameter), there are important methodologic aspects to consider when interpreting and comparing results from different investigations. In some studies, relatively thick sections of 3-5 mm have been used. ${ }^{9,12,13,29}$ Thick sections increase the partial volume effect from high-signal-intensity tissues like bone marrow and fatty tissues and thus probably contribute to high-signal-intensity areas seen in the ligaments. In the present study, thin sections $(2 \mathrm{~mm})$ in 3 different imaging planes were used to avoid partial volume effects from the surrounding tissues. However, even with thin sections and high in-plane resolution, the interpretation of signal-intensity changes is difficult.

Another aspect is the influence of the magnetic field strength on the image quality and the consequential ability to detect small structural changes in the ligaments. In some studies, a low-field magnetic strength $(0.2-0.5 \mathrm{~T})$ was used, 9,10,12,13,29 whereas other investigators used a high-field magnetic strength (1.5T). ${ }^{15,30}$ In the present study, we used a magnetic field strength of $1.5 \mathrm{~T}$. Another variable that probably influences the wide range of imaging results is the choice of MR imaging parameters. In some studies, both T1 and T2 images have been obtained. ${ }^{9,13,15,29,30}$ An early study of Krakenes et $\mathrm{al}^{14}$ investigated various MR imaging parameters and concluded that detailed structural information of ligaments and membranes in the craniocervical region was best obtained with high-resolution proton attenuation-weighted images in 3 planes. This type of imaging sequence was also applied in the present study.

Another difficulty when interpreting signal intensity of alar ligaments on MR imaging is the grading of signal-intensity hyperintensities. Krakenes et $\mathrm{al}^{15}$ introduced a 4 -point grading scale based on the signal intensity of the alar ligaments in the sagittal plane. The same 4-point grading technique was used in subsequent studies ${ }^{9,14,27}$ as well as in this study. Nevertheless, the low inter-rater agreement rates concerning signal intensity in the present and in previous studies ${ }^{11}$ indicate that using this 4-point grading scale may have disadvantages in clinical prac- tice. Even after dichotomization, the inter- and intrarater $\kappa$ values of the groups did not have an acceptable agreement for this rating scale to be clinically useful. An erroneous evaluation of 1 or both readers as another possible explanation for the low inter-rater agreement seems unlikely considering the very good interobserver agreement concerning the orientation of the alar ligaments.

To which degree alar ligaments are really affected in WAD is not even completely clear yet. ${ }^{7}$ One of the few postmortem studies showed that injury of the alar ligaments is possible in the context of heavy trauma. ${ }^{4}$ In 11 of 30 specimens with various extent of head trauma (from subgaleal hemorrhage to comminuted skull fracture), rupture or underbleeding of alar ligaments was found, but there was no correlation between the degree of trauma and the extent of injury of the ligaments. In 2008 , a second postmortem investigation was published. ${ }^{28}$ As mentioned above, $\operatorname{Koch}^{28}$ examined human specimens after fatal head trauma by using MR imaging and subsequent macroscopic and microscopic correlation. Even though 13 of 14 specimens showed severe injury of the upper cervical spine, involvement of the alar ligament, meaning elongation or partial or complete rupture, was not found in any of the specimens.

Furthermore, other biomechanical experiments with special focus on injuries of the upper cervical spine and craniocervical junction could not verify injuries of the alar ligaments. ${ }^{31-33}$ Nevertheless, these experiments can only be an approach to reality because the influence of muscular tonicity, for example, cannot be simulated. Results of animal studies in which transient pressure pulses in the cervical spinal canal during experimental neck trauma were recorded indicate that WAD could also appear due to other factors, such as involvement of the cervical spinal nerve root region. ${ }^{34}$ It was hypothesized that the changes in the inner volume of the cervical spinal canal during neck extension-flexion motion would cause transient pressure changes in the central nervous system as a result of hydrodynamic effects and thereby mechanically load the nerve roots and cause tissue damage. However, this hypothesis and the transferability to WAD in humans need further evaluation.

There are some limitations to the present study. First, the average age of the population in this investigation was fairly young with a limited age range. To evaluate a correlation between hyperintense signal intensity of the alar ligaments and interspersed fatty tissue due to degeneration, a study should correlate signal intensity with age. In addition, histopathologic correlations would be helpful, but specimens of patients with low-grade WAD are certainly rare. Second, we did not include a control group with WAD in this investigation. Hence a potential difference concerning morphology or signal intensity of the alar ligaments could not be evaluated. Furthermore longitudinal studies including an MR imaging investigation before and after WAD would give revealing information on this contradictory topic.

\section{Conclusions}

Our study and previous reports show that alar ligaments can be clearly detected by using high-field MR imaging. In patients with acute trauma, MR imaging can rule out structural pathologies of the alar ligaments, which are indeed rarely found. 
Nevertheless, the benefit of imaging alar ligaments for the diagnosis of chronic low-grade WAD is contestable in consideration of the ligaments' remarkable variability of morphology, course, and signal intensity. Given our current results and the data, we advise against interpreting areas of high intensity in alar ligaments on MR imaging as a morphologic correlate to WAD. Our results do not support any diagnostic value or clinical relevance of these signal-intensity hyperintensities.

\section{References}

1. Standring S. Gray's Anatomy: The Anatomical Basis of Medicine and Surgery. $39^{\text {th }}$ ed. New York: Churchill-Livingstone; 2004

2. Dvorak J, Panjabi MM. Functional anatomy of the alar ligaments. Spine 1987;12:183-89

3. Crisco JJ 3rd, Panjabi MM, Dvorak J. A model of the alar ligaments of the upper cervical spine in axial rotation. J Biomech 1991;24:607-14

4. Saternus KS, Thrun C. Traumatology of the alar ligaments. Aktuelle Traumatol 1987; 17:214-18

5. Dickman CA, Greene KA, Sonntag VK. Injuries involving the transverse atlantal ligament: classification and treatment guidelines based upon experience with 39 injuries. Neurosurgery 1996;38:44-50

6. Fielding JW, Cochran GB, Lawsing JF 3rd, et al. Tears of the transverse ligament of the atlas: a clinical and biomechanical study. J Bone Joint Surg Am 1974;56:1683-91

7. Bitterling $H$, Stabler A, Bruckmann H. Mystery of alar ligament rupture: value of MRI in whiplash injuries - biomechanical, anatomical and clinical studies [in German]. Rofo 2007;179:1127-36

8. Krakenes J, Kaale BR. Magnetic resonance imaging assessment of craniovertebral ligaments and membranes after whiplash trauma. Spine 2006; 31:2820-26

9. Pfirrmann CW, Binkert CA, Zanetti M, et al. MR morphology of alar ligaments and occipitoatlantoaxial joints: study in $\mathbf{5 0}$ asymptomatic subjects. Radiology 2001;218:133-37

10. Willauschus WG, Kladny B, Beyer WF, et al. Lesions of the alar ligaments: in vivo and in vitro studies with magnetic resonance imaging. Spine 1995;20:2493-98

11. Myran R, Kvistad KA, Nygaard OP, et al. Magnetic resonance imaging assessment of the alar ligaments in whiplash injuries: a case-control study. Spine 2008;33:2012-16

12. Wilmink JT, Patijn J. MR imaging of alar ligament in whiplash-associated disorders: an observer study. Neuroradiology 2001;43:859-63

13. Roy S, Hol PK, Laerum LT, et al. Pitfalls of magnetic resonance imaging of alar ligament. Neuroradiology 2004;46:392-98. Epub 2004 Apr 27

14. Krakenes J, Kaale BR, Rorvik J, et al. MRI assessment of normal ligamentous structures in the craniovertebral junction. Neuroradiology 2001;43:1089-97

15. Krakenes J, Kaale BR, Moen G, et al. MRI assessment of the alar ligaments in the late stage of whiplash injury: a study of structural abnormalities and observer agreement. Neuroradiology 2002;44:617-24

16. Beschleunigungstrauma der Halswirbelsäule: Leitlinien für Diagnostik und Therapie in der Neurologie. 4th ed. Stuttgart: Georg Thieme Vg; 2008

17. Chappuis G, Soltermann B, CEA, et al. Number and cost of claims linked to minor cervical trauma in Europe: results from the comparative study by CEA, AREDOC, and CEREDOC. Eur Spine J 2008;17:1350-57. Epub 2008 Aug 15

18. Spitzer WO, Skovron ML, Salmi LR, et al. Scientific monograph of the Quebec Task Force on Whiplash-Associated Disorders: redefining "whiplash" and its management. Spine (Phila Pa 1976) 1995;20(8 suppl):1S-73S

19. Cohen J. A coefficient of agreement for nominal scales. Educ Psychol Meas 1960;20:213-20

20. Kaale BR, Krakenes J, Albrektsen G, et al. Clinical assessment techniques for detecting ligament and membrane injuries in the upper cervical spine region: a comparison with MRI results. Man Ther 2008;13:397-403

21. Daniels DL, Williams AL, Haughton VM. Computed tomography of the articulations and ligaments at the occipito-atlantoaxial region. Radiology 1983;146:709-16

22. Panjabi MM, Oxland TR, Parks EH. Quantitative anatomy of cervical spine ligaments. Part I. Upper cervical spine. J Spinal Disord 1991;4:270-76

23. el-Khoury GY, Wira RL, Berbaum KS, et al. MR imaging of patellar tendinitis. Radiology 1992;184:849-54

24. Hodler J, Haghighi P, Trudell D, et al. The cruciate ligaments of the knee: correlation between MR appearance and gross and histologic findings in cadaveric specimens. AJR Am J Roentgenol 1992;159:357-60

25. Kjellin I, Ho CP, Cervilla V, et al. Alterations in the supraspinatus tendon at MR imaging: correlation with histopathologic findings in cadavers. Radiology 1991;181:837-41

26. Schweitzer ME, Mitchell DG, Ehrlich SM. The patellar tendon: thickening, internal signal buckling, and other MR variants. Skeletal Radiol 1993;22: 411-16

27. Vetti N, Krakenes J, Eide GE, et al. MRI of the alar and transverse ligaments in whiplash-associated disorders (WAD) grades 1-2: high-signal changes by age, gender, event and time since trauma. Neuroradiology 2009;51:227-35

28. Koch S. Magnetresonanztomographische, pathoanatomische und histologische Korrelation von Ligamenta alaria an humanen Präparaten nach gewaltsamem Tod [Dissertation]. Munich, Germany: Ludwig Maximilian University of Munich;2008

29. Volle E, Montazem A. MRI video diagnosis and surgical therapy of soft tissue trauma to the craniocervical junction. Ear Nose Throat J 2001;80:41-44, 46-48

30. Kim HJ, Jun BY, Kim WH, et al. MR imaging of the alar ligament: morphologic changes during axial rotation of the head in asymptomatic young adults. Skeletal Radiol 2002;31:637-42

31. Panjabi MM, Cholewicki J, Nibu K, et al. Biomechanics of whiplash injury [in German]. Orthopade 1998;27:813-19

32. Panjabi MM, Cholewicki J, Nibu K, et al. Mechanism of whiplash injury. Clin Biomech (Bristol, Avon) 1998;13:239-49

33. Panjabi MM, Cholewicki J, Nibu K, et al. Simulation of whiplash trauma using whole cervical spine specimens. Spine 1998;23:17-24

34. Svensson MY, Aldman B, Bostrom O, et al. Nerve cell damages in whiplash injuries: animal experimental studies [in German]. Orthopade 1998;27:820-26 